\title{
Bispectrum-based Cross-frequency Functional Connectivity: A Study of Alzheimer's Disease
}

\author{
Dominik Klepl, ${ }^{1}$ Fei He, ${ }^{1}$, ${ }^{\text {Min }} \mathrm{Wu},{ }^{2}$ Daniel J. Blackburn, ${ }^{3}$ and Ptolemaios G. Sarrigiannis ${ }^{4}$ \\ ${ }^{1}$ Centre for Computational Science and Mathematical Modelling, Coventry University, Coventry CV1 2JH, UK \\ ${ }^{2}$ Institute for Infocomm Research, Agency for Science, \\ Technology and Research (A*STAR), 138632, Singapore \\ ${ }^{3}$ Department of Neuroscience, University of Sheffield, Sheffield, S10 2HQ, UK \\ ${ }^{4}$ Department of Neurophysiology, Royal Devon and Exeter NHS Foundation Trust, Exeter, EX2 5DW, UK
}

\begin{abstract}
Alzheimer's disease (AD) is a neurodegenerative disorder known to affect functional connectivity (FC) across many brain regions. Linear FC measures have been applied to study the differences in AD by splitting neurophysiological signals such as electroencephalography (EEG) recordings into discrete frequency bands and analysing them in isolation from each other. We address this limitation by quantifying cross-frequency FC in addition to the traditional within-band approach. Crossbispectrum, a higher-order spectral analysis approach, is used to measure the nonlinear FC and is compared with the cross-spectrum, which only measures the linear FC within bands. This work reports the first use of cross-bispectrum to reconstruct a cross-frequency FC network where each frequency band is treated as a layer in a multilayer network with both inter- and intra-layer edges. An increase of within-band FC in $\mathrm{AD}$ is observed in low-frequency bands using both methods. Bispectrum also detects multiple cross-frequency differences, mainly increased FC in AD in delta-theta coupling. An increased importance of low-frequency coupling and decreased importance of highfrequency coupling is observed in $\mathrm{AD}$. Integration properties of $\mathrm{AD}$ networks are more vulnerable than $\mathrm{HC}$, while the segregation property is maintained in AD. Moreover, the segregation property of $\gamma$ is less vulnerable in $\mathrm{AD}$, suggesting the shift of importance from high-frequency activity towards low-frequency components. The results highlight the importance of studying nonlinearity and including cross-frequency $\mathrm{FC}$ in characterising $\mathrm{AD}$. Moreover, the results demonstrate the advantages and limitations of using bispectrum to reconstruct FC networks.
\end{abstract}

\section{INTRODUCTION}

Alzheimer's disease (AD) causes early degradation of neural circuits leading to cell death and synaptic loss and is the most common form of dementia [1, 2]. Studies have shown that AD affects distributed brain networks, alters functional connectivity, which can lead to disconnection syndrome and disrupts information processing across multiple scales [3] 6 .

Electroencephalography (EEG) is a common method to study and diagnose AD. The main EEG characteristics associated with AD are slowing of signals and decreased synchronisation [4, 6, 9]. Slowing of EEG in AD was observed as increased activity in $\delta$ and $\theta$ frequency bands and decreased activity in $\alpha$ and $\beta$ frequency bands [6, 7]. Similarly, AD shows changes in synchronisation within low-frequency bands $(<12 \mathrm{~Hz})$ and is associated with the altered functional connectivity, especially the long-distance cortical connections [10. A recent focus on network-based methods reveals additional characteristics of $\mathrm{AD}$, namely, reduced integration of information [11, 12 and loss of small-worldness [13]. However, these characteristics are typically analysed only within specific frequency bands.

This study aims to extend the functional connectivity beyond within-frequency connectivity (WFC) and take the cross-frequency coupling (CFC) [14] into account.

\footnotetext{
* Correspondence to: fei.he@coventry.ac.uk
}

WFC connectivity was previously analysed in AD by using coherence (linear) [15] and wavelet coherence (nonlinear) [16]. Only one CFC measure, i.e. phase synchronisation index (PSI), had been used to reconstruct CFC networks of AD [17]. This work extended the findings of reduced integration and loss of small-worldness to CFC multilayer networks. However, it does not consider the roles of different frequency components in the networks, and PSI is purely a phase-phase coupling measure. Other attempts to use multilayer networks to characterise neurological disorders were made. Guillon et al. [18 reported loss of inter-frequency hubs in AD using MEG multilayer networks, but without explicit inclusion of CFC, rather inter-layer (i.e. cross-frequency) connections were made only between the same nodes across layers. A similar approach was used to characterise schizophrenia from MEG [19].

Bispectrum is a higher-order extension of the spectrum and quantifies quadratic coupling between two frequency components and their algebraic sum 20. It can detect amplitude-amplitude and phase-amplitude CFC in addition to phase-phase coupling [21. The bispectral coupling also indicates an increase in non-Gaussianity [22]. Features derived from bispectrum were shown to provide biomarkers of epilepsy [23], Parkinson's disease [24] and AD [22]. These studies compute (cross-) bispectra of only a few channels or pairs of channels. In contrast, this study computes cross-bispectra between all pairs of EEG channels to estimate the whole-brain FC network.

In this work, the cross-bispectrum (CBS) estimates 

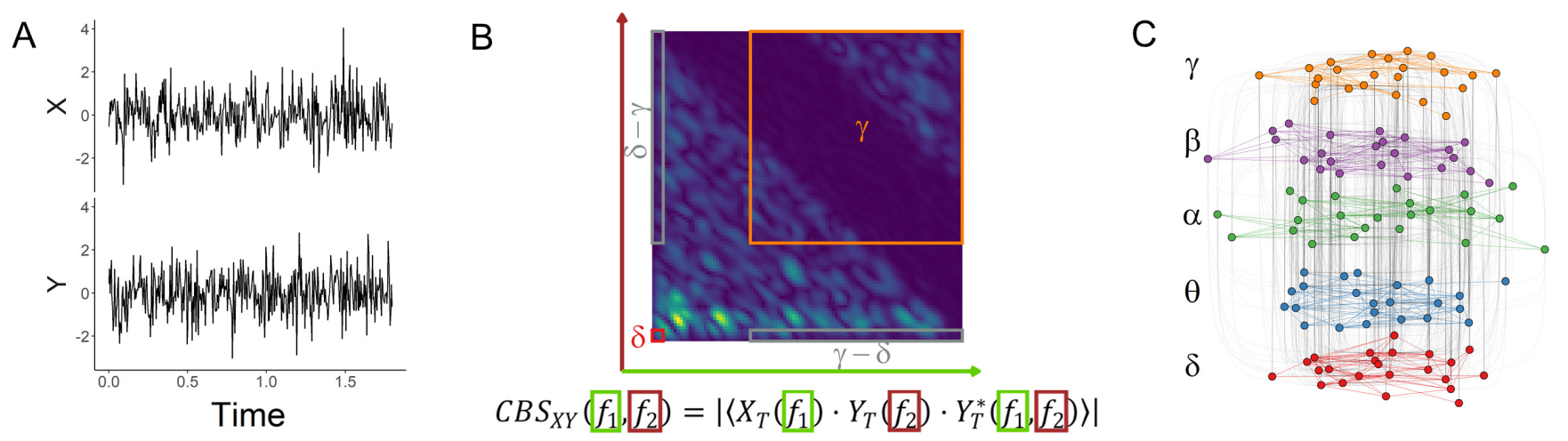

FIG. 1: A conceptual schematic of implementing the proposed cross-bispectrum (CBS) multilayer network analysis. (A) Each EEG signal is cleaned and scaled. (B) For each pair of EEG electrodes, a cross-bispectrum is estimated. The frequency bands coupling edge weights are given by the maximum value within the respective CBS window, e.g. $\delta$ - $\delta$ (red). Note that CBS estimates are directed, e.g. $\delta-\gamma \neq \gamma-\delta$ (both in black). Thus from each CBS, 25 edges are inferred. (C) Using the edge weights inferred from CBS, a multilayer network is constructed with layers representing the frequency bands of EEG. Such a network has both intra-layer edges and inter-layer edges, representing the within-frequency coupling and cross-frequency coupling, respectively.

of functional connectivity are computed in order to investigate the contribution of nonlinear CFC to the differences between Alzheimer's disease (AD) and healthy controls (HC) compared to linear WFC measured with cross-spectrum (CS). To the best of our knowledge, this is the first application of CBS to reconstruct FC networks of AD. Moreover, we report a novel multilayer-networkbased approach to elucidate the roles of the traditional EEG frequency bands and their CFC in the whole-brain networks of $\mathrm{HC}$ and $\mathrm{AD}$.

\section{DATA}

EEG recordings were collected from $20 \mathrm{AD}$ patients and 20 healthy participants (HC) under 70. A detailed description of the experimental design and confirmation of the diagnosis is provided in 25. All AD participants were recruited in the Sheffield Teaching Hospital memory clinic. AD participants were diagnosed between 1 month and 2 years before data collection, and all of them were in the mild to moderate stage of the disease at the time of recording with the average Mini Mental State Examination score of $20.1(s d=4)$. High resolution structural magnetic resonance imaging (MRI) scans of all patients were acquired to eliminate alternative causes of dementia. Age and gender-matched HC participants with normal neuropsychological tests and structural MRI scans were recruited.

EEG was acquired using an XLTEK 128-channel headbox, $\mathrm{Ag} / \mathrm{AgCL}$ electrodes with a sampling frequency of $2 \mathrm{kHz}$ using a modified 10-10 overlapping and 10-20 international electrode placement system with a referential montage with a linked earlobe reference. The recordings lasted 30 minutes, during which the participants were instructed to rest and not to think about anything specific. Within the 30 minutes recording, there were two-minute- long epochs during which the participants had their eyes closed (EC) (alternating with eyes-open epochs which are not used in this study).

All the recordings were reviewed by an experienced neurophysiologist on the XLTEK review station with time-locked video recordings (Optima Medical LTD). For each participant, three 12-second-long artefact-free epochs were isolated. Finally, to avoid volume conduction effects related to the common reference electrodes, the following 23 bipolar channels were created: F8-F4, F7-F3, F4-C4, F3-C3, F4-FZ, FZ-CZ, F3-FZ, T4-C4, T3-C3, C4-CZ, C3-CZ, CZ-PZ, C4-P4, C3-P3, T4-T6, T3-T5, P4-PZ, P3-PZ, T6-O2, T5-O1, P4-O2, P3-O1 and $\mathrm{O} 1-\mathrm{O} 2$.

\section{A. EEG pre-processing}

EEG signals were filtered to be between 0.5 and 100 $\mathrm{Hz}$ using Fourier transform; $50 \mathrm{~Hz}$ relating to the power line noise was removed and the data were downsampled to $250 \mathrm{~Hz}$. Only the first 4 seconds of each epoch were used. Finally, the signals were normalised (to zero mean and unit standard deviation).

\section{METHODS}

\section{A. Cross-spectrum and cross-bispectrum}

The spectrum of signal $S_{X}$ is calculated via smoothed periodogram. Fast Fourier Transform (FFT) is used to estimate the periodogram with Daniell smoothers. The periodogram is computed over 256 frequency bins (0.98 $\mathrm{Hz}$ bandwidth). CS at frequency $f$ is then computed as: $C S_{X Y}(f)=S_{X}(f) \cdot S_{Y}(f)$. An absolute value of 
A

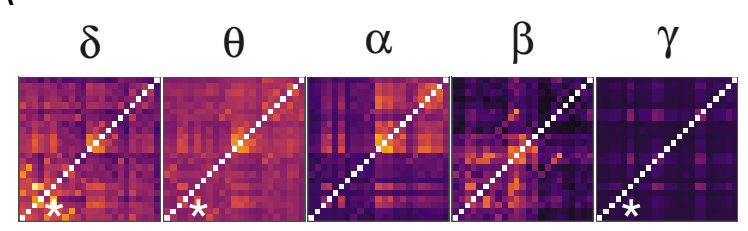

B

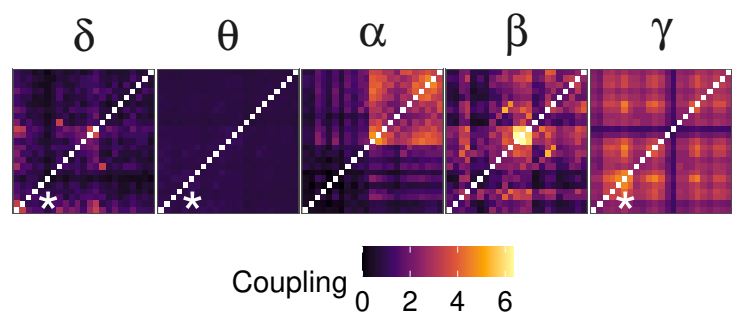

FIG. 2: Average connectivity matrices measured with cross-spectrum of (A) AD and (B) HC. For visualisation purposes, the values were normalised. White asterisks denote a significant difference in global coupling $(p<0.05)$.

CS is calculated. A direct FFT-based method is used to estimate absolute CBS:

$$
C B S_{X Y}\left(f_{1}, f_{2}\right)=\left|\left\langle X_{T}\left(f_{1}\right) \cdot Y_{T}\left(f_{2}\right) \cdot Y_{T}^{*}\left(f_{1}, f_{2}\right)\right\rangle\right|,
$$

where $\langle\cdot\rangle$ denotes averaging, $X_{T}(f)$ is a Fourier Transform of signal $X$ over an interval $\mathrm{T}$ and $Y^{*}$ is the complex conjugate. 256-point FFT is used. CBS is computed over $0.08 \mathrm{~s}$ long segments with $50 \%$ overlap over whole frequency range $(0.5-100 \mathrm{~Hz})$. The estimated CBS is then smoothed in the frequency domain using Rao-Gabr window (size 5).

CS and CBS were computed for all pairs of EEG channels. Five frequency bands $b$ are considered: $\delta$ $(0.5-4 H z), \theta(5-7 H z), \alpha(8-15 H z), \beta(16-31 H z)$ and $\gamma(32-100 \mathrm{~Hz})$.

The connectivity $C N$ between channels $X$ and $Y$ and frequency bands $b_{X}$ and $b_{Y}$ is computed as:

$$
\begin{gathered}
C N_{X Y}^{C S}\left(b_{X}\right)=\max \left(C S_{X Y}\left(f \in b_{X}\right)\right), b_{X}=b_{Y} \\
C N_{X Y}^{C B S}\left(b_{X}, b_{Y}\right)=\max \left(C B S_{X Y}\left(f_{1} \in b_{X}, f_{2} \in b_{Y}\right)\right),
\end{gathered}
$$

for $\mathrm{CS}$ and CBS respectively. This resulted in $5 \mathrm{WFC}$ (CS and CBS) and 20 CFC (CBS only) measures for each pair of channels. It is of note that the CBS is directed.

In order to ensure statistical significance of the estimated connectivity, surrogate thresholding was used [26]. For each pair of channels, 20 surrogate signals were generated with phase randomly shuffled, and their connectivity is computed. The mean of surrogate connectivity is used as a threshold. Connectivity values below the threshold are set to zero.

We obtain a set of connectivity matrices for each EEG recording, i.e. $N \times N$ matrices $(N=23)$. For $\mathrm{CS}$ and
CBS, there are 5 and 25 connectivity matrices, respectively. A global (averaged per subject) connectivity is computed for each $23 \times 23$ matrix and compared between groups using Mann-Whitney test.

\section{B. Network measures}

To identify the important channels in the network, we compute a coupling specific node strength (NS) [27] for each channel $i$ and the different types of frequency couplings $c$ given by:

$$
N S(i, c)=\sum_{j \in \Pi(i, c)} w_{i j}
$$

where $\Pi(i, c)$ are the nodes connected to $i$ by edge type $c$ and $w_{i j}$ is the edge weight, i.e. CS or CBS connectivity given by $i j$ th entry of $N \times N$ connectivity matrix. This measure is computed both for CS and CBS, resulting in 5 ( 5 frequency bands) and $25(5 \times 5$ frequency bands) values per channel respectively.

In order to analyse the importance of the different frequency couplings in terms of the whole brain network, we represent them in a multilayer network. In this network, nodes are located within layers representing the different frequency bands, WFC represents the edges between nodes within a single layer, i.e. intra-layer, and CFC represents the edges between nodes located in different layers, i.e. inter-layer. In this paper, the CS networks are not analysed as multilayer networks since such networks would have no inter-layer edges and thus would not be directly comparable with the CBS networks. The following measures are computed only for CBS networks. We obtain networks with 23 nodes that are replicated over 5 layers $(L \in[\delta, \theta, \alpha, \beta, \gamma])$, resulting effectively in 115 nodes. There are 5 types of intra-layer edges such as $\delta-\delta$, and 20 types of inter-layer edges such as $\delta-\theta$ or $\theta-\delta$. Moreover, as the following graph-theoretic measures assume that the edge weight represents a distance, we transform the weights as $1 / w_{i j}$ so that a strong coupling value results in a low weight.

We measure the importance of each type of frequency coupling within the multilayer network by measuring the contribution of each edge to enabling efficient passing of information through the network. For this purpose, we define betweenness centrality (CBW) based on an adjusted version of edge betweenness (BW) [28]:

$$
C B W(c)=\frac{1}{E} \sum_{i=1}^{E} B W(e),
$$

where $E$ is the total number of edges of coupling type $c$ and $B W(e)$ is edge betweenness centrality given by:

$$
B W(e)=\sum_{i \neq j} \frac{g_{i j}(e)}{g_{i j}},
$$


bioRxiv preprint doi: https://doi.org/10.1101/2021.08.07.455499; this version posted August 8, 2021. The copyright holder for this preprint (which was not certified by peer review) is the author/funder, who has granted bioRxiv a license to display the preprint in perpetuity. It is made available under aCC-BY-NC 4.0 International license.

A

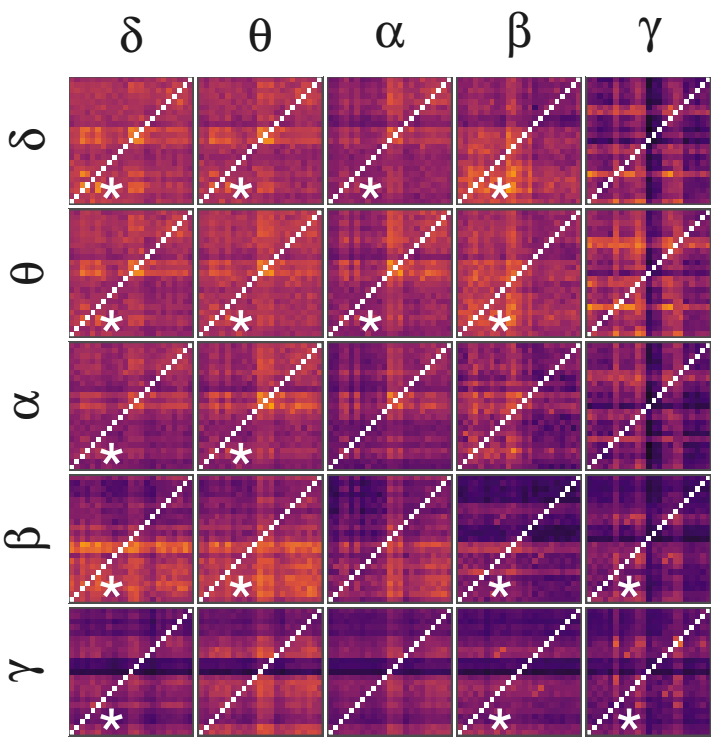

$\mathrm{B}$

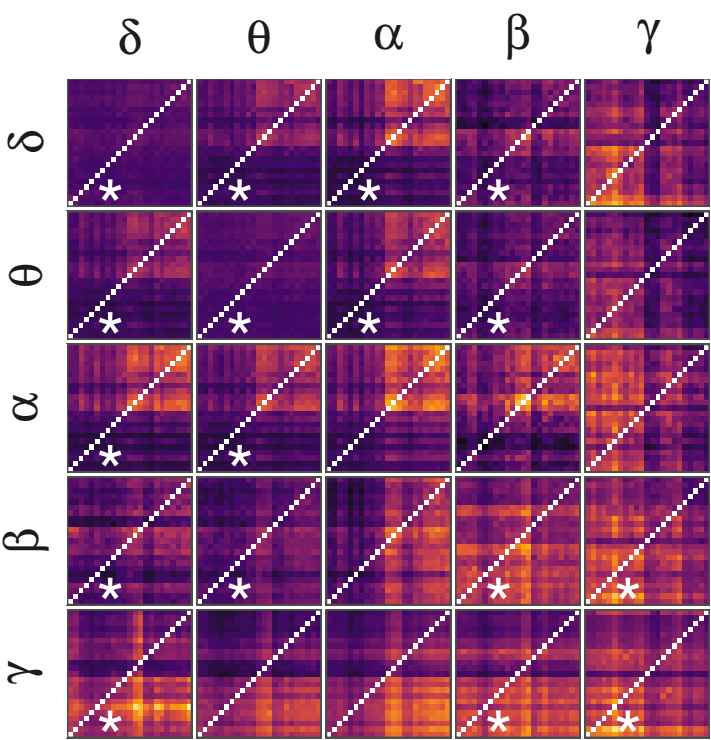

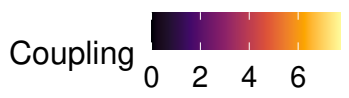

FIG. 3: Average connectivity matrices measured with cross-bispectrum of (A) AD and (B) HC with input frequency on the vertical facets and output frequency on horizontal. For visualisation purposes, the values were normalised. White asterisks denote a significant difference in global coupling $(p<0.05)$.

where $g_{i j}$ is the number of shortest paths between nodes $i$ and $j$, and $g_{i j}(e)$ is the number of those paths that go through edge $e$. CBW quantifies the contribution of each coupling type to the information integration [29], i.e. the amount of information flow through edges.

CBW assumes that the essential processes within the network occur along the shortest paths. However, there might be alternative paths with only minor length differences, which CBW ignores. In case of a disruption of the network structure, these alternative paths might enable recovery of function with negligible differences. We quantify this as the vulnerability of the network to the removal of one type of frequency coupling. The vulnerability is measured in two ways, the loss of ability to integrate information [30] and the loss of segregation.

The integration property of network $G$, i.e. the ability of a network to communicate information globally, is approximated with global efficiency ( $\mathrm{gE}$ ) given by:

$$
g E(G)=\frac{1}{N(N-1)} \sum_{i \neq j \in G} \frac{1}{d(i j)}
$$

where $N$ is the number of nodes in network $G$ and $d(i j)$ is the shortest path length between nodes $i$ and $j$. $\mathrm{gE}$ is related to CBW. CBW measures the information flow on the more detailed edge-level while $\mathrm{gE}$ takes the node-level perspective.

The segregation property of network $G$, i.e. the presence of densely connected clusters and sparse connections between them, is approximated with local efficiency given by:

$$
l E(G)=\frac{1}{N} \sum_{i \in G} g E\left(G_{i}\right)
$$

where $G_{i}$ is the neighbourhood of node $i$, i.e. subgraph of nodes directly connected to $i$, without node $i$ itself.

In order to measure the vulnerability of the network and its dependence on different types of frequency coupling $g E$ and $l E$ is computed for the full network, the two measures are re-computed on a perturbed network with single type of frequency coupling (i.e. set of edges) removed and the change in $g E$ and $l E$ is computed, resulting in the vulnerability measures $g V\left(G_{c}\right)=1-$ $\left(g E\left(G_{c}\right) / g E(G)\right)$ and $l V=1-\left(l E\left(G_{c}\right) / l E(G)\right)$, where $G$ is the full network and $G_{c}$ is the perturbed network.

\section{Network thresholding and statistical analysis}

In order to filter out the unimportant edges that might be the result of a spurious coupling, the weighted multilayer networks are thresholded through relative quantilebased thresholding. Given a quantile $Q$, all edges with weight lower than $Q$ are removed from the network. There are large differences between the weights of each frequency coupling type (e.g. mean of $\gamma-\beta=1.627 \mathrm{com}-$ pared to mean of $\alpha-\alpha=8.975)$; thus, a separate thresh- 


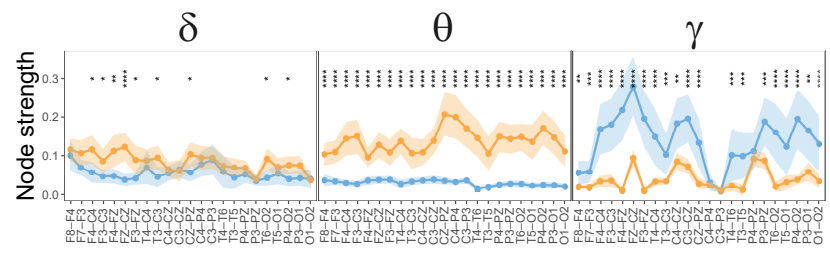

FIG. 4: Node strength (normalised) measured with CS of $\mathrm{HC}$ (blue) and AD (orange): mean with $95 \%$ confidence intervals. Significant differences showed as: $\mathrm{p} \leq 0.05\left(^{*}\right), \mathrm{p}$ $\leq 0.01\left(^{* *}\right), \mathrm{p} \leq 0.001\left(^{* * *}\right), \mathrm{p} \leq 0.0001(* * * *)$.

old $Q$ is used. As a result, the networks retain $Q \%$ of the strongest edges. To ensure that the observed differences between the networks are not due to the choice of threshold $Q$, all of the network measures are computed over 10 of threshold values $(Q \in[0.5,0.95]$ in increments of 0.05 ) and only significant differences observed over at least 3 consecutive thresholds are declared significant. The reported plots and numerical results are obtained with $Q=0.7$. Additionally, we report the number of consecutive thresholds where significant differences were observed. All p-values are corrected using the false discovery rate method.

As NS compares the different frequency coupling types in isolation, the weighted networks are used in this analysis. Furthermore, we convert the network from directed to undirected by taking the maximum weight for each pair of directed edges, thus collapsing them into a single edge. Since NS follows a Gamma distribution, the Gamma generalised linear model (GGLM) with log-link function is used to compare the groups. Note that NS is computed both for CS and CBS networks and frequency bands that show a significant difference in the global averaged coupling strength.

The multilayer graph measures aim to analyse the roles of the frequency coupling types in the properties of the global network. Since differences were observed between the weights of frequency coupling types, we convert the weighted networks into unweighted (or binary) networks by setting the weight of all edges to 1 , thus removing the potential effect of the observed differences. Both $C B W$ and $l V$ are not normally distributed but symmetrical around the median. Thus, a non-parametric MannWhitney test is used to compare between groups. As $g V$ is Gamma-distributed, GGLM with log-link function is used to compare between the groups.

\section{RESULTS AND DISCUSSION}

\section{A. Connectivity matrices and global connectivity}

Averaged connectivity matrices (Fig. 2 and 3 indicate that both methods seem to detect differences in the topology of functional connectivity networks. In CS, these differences seem to be the most pronounced in $\delta$ and $\theta$ where AD have higher connectivity and in $\gamma$ where $\mathrm{AD}$ have lower connectivity, as shown by the differences in global connectivity. In CBS, differences can be observed in almost all frequency bands and their couplings. The topology differences are rather obvious in $\delta, \theta, \alpha$ and their CFC couplings wherein possible clusters are visible in $\mathrm{HC}$, while $\mathrm{AD}$ seem to have this structure distorted. Moreover, significant differences were found in global connectivity in all $\delta, \theta$ and $\beta$ CFC (except for $\delta$ $\gamma, \theta-\gamma$ and $\beta-\alpha)$ and in $\beta-\beta$. Differences in these bands (both WFC and CFC) were reported by Cai et al. [17] using PSI, but the reported differences are opposite to our findings. Moreover, we found significant differences in $\gamma \mathrm{WFC}$ and $\gamma-\delta$ and $\gamma-\beta$ CFC.

These findings fit well with the literature reporting the increased activity in $\delta$ and $\theta$ in $\mathrm{AD}$ [4, 6. Moreover, the visible distortion of structure within multiple frequency bands detected both by CS and CBS is in line with the disconnection syndrome and disturbed information processing in AD.

\section{B. Coupling-wise node strength}

In order to statistically test the differences in connectivity measured by both CS and CBS and to localise the brain regions which show the most pronounced differences between $\mathrm{AD}$ and $\mathrm{HC}$, NS is measured for each channel and coupling type separately. Due to the space limits, we do not report the numerical results of these tests. Alternatively, we show the results in Figs. 2 and 3 for $\mathrm{CS}$ and $\mathrm{CBS}$ respectively.

The differences in WFC detected by CS and CBS (Fig. 4 and diagonal elements in Fig. 5) are generally similar. Both methods show increased $\delta$ and $\theta$ NS in AD across most channels. CS shows increased $\gamma \mathrm{NS}$ in $\mathrm{HC}$ in frontal and temporal channels. In addition, CBS detected differences in $\beta$ and $\gamma \mathrm{WFC}$ in a few central and temporal channels, which CS missed. These differences showcase the importance of assessing both linear and nonlinear coupling to understand the changes in $\mathrm{AD}$ brain networks.

Multiple differences in CFC (off-diagonal elements in Fig. 5) were detected, highlighting the need to analyse the relationships between frequency components both in healthy and AD brain networks. Interestingly, the CFC involving $\delta$ and $\theta$ seems to be the most affected by $\mathrm{AD}$, extending the findings in the literature (i.e. AD showing increased activity in these bands) from WFC to CFC as well, as the $\delta-\theta$ and $\theta-\delta$ shows increased NS in AD in frontal and a few central, temporal and parietal channels. Predominantly frontal channels show increased NS in $\mathrm{AD}$ in $\delta-\alpha, \theta-\alpha$ and their inverse, i.e. $\alpha-\delta$ and $\alpha-\theta$. This increased NS in AD is also present in a few frontal channels in $\delta-\beta, \theta-\beta$ and their inverse.

Cai et al. [17] report comparable differences using PSI between $\mathrm{AD}$ and $\mathrm{HC}$, but in contrast to our results, they report mainly decreased NS in AD. This might be be- 
bioRxiv preprint doi: https://doi.org/10.1101/2021.08.07.455499; this version posted August 8, 2021. The copyright holder for this preprint (which was not certified by peer review) is the author/funder, who has granted bioRxiv a license to display the preprint in perpetuity. It is made available under aCC-BY-NC 4.0 International license.
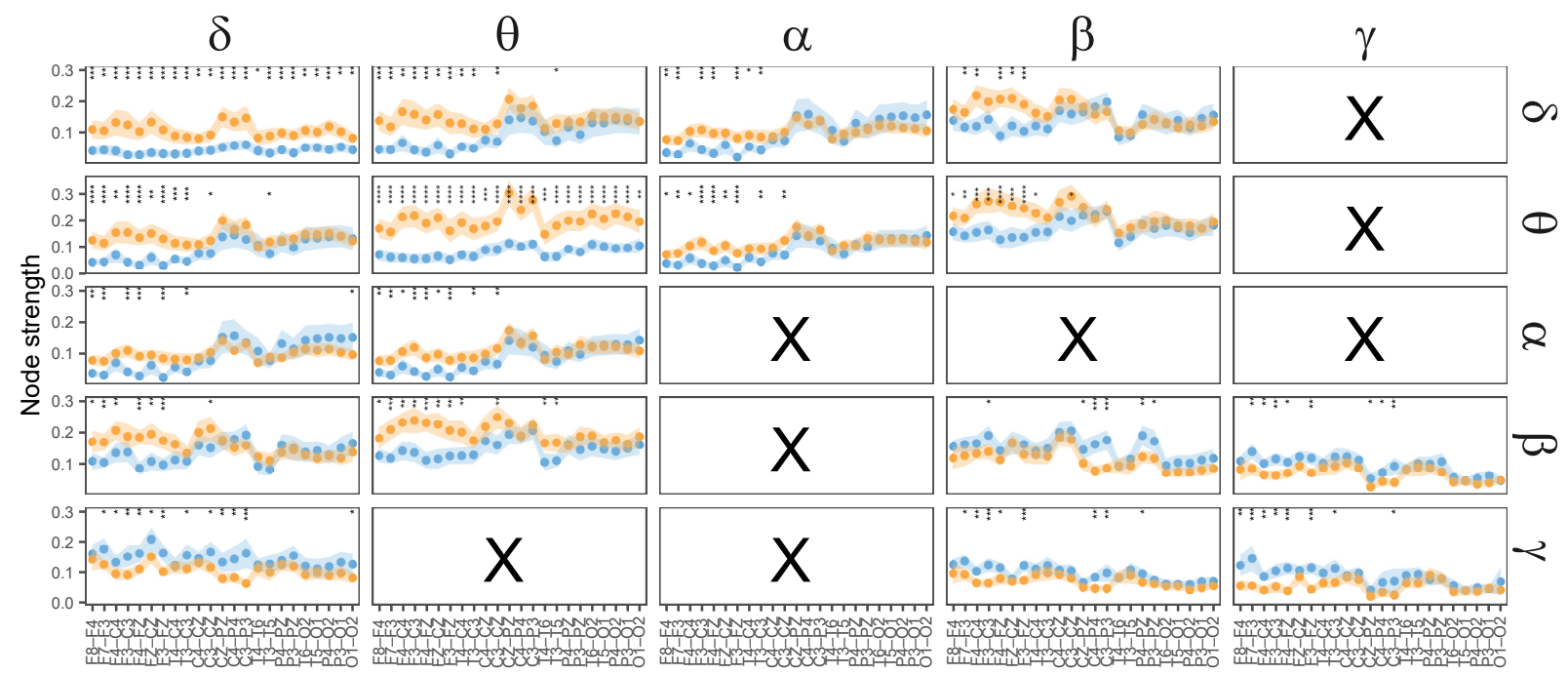

FIG. 5: Node strength (normalised) measured with CBS of HC (blue) and AD (orange): mean with 95\% confidence intervals. Input frequency is on the vertical facets and output frequency on the horizontal. Significant differences are showed as: $\mathrm{p}$ $\leq 0.05(*), \mathrm{p} \leq 0.01(* *), \mathrm{p} \leq 0.001(* * *), \mathrm{p} \leq 0.0001(* * * *)$.

\begin{tabular}{|c|c|c|c|c|c|}
\hline & $\delta$ & $\theta$ & $\alpha$ & $\beta$ & $\gamma$ \\
\hline & CBW: $\mathrm{U}=946, \mathbf{p}<\mathbf{0 . 0 0 1}, 10$ & CBW: $\mathrm{U}=882, \mathbf{p}<\mathbf{0 . 0 0 1}, 10$ & CBW: $\mathrm{U}=834, \mathbf{p}<\mathbf{0 . 0 0 1}, 10$ & CBW: $\mathrm{U}=1670, \mathrm{p}=0.604,0$ & CBW: $\mathrm{U}=2391, \mathbf{p}=\mathbf{0 . 0 0 2}, 8$ \\
\hline & $\mathrm{gV}: \mathrm{t}=4.632, \mathbf{p}<\mathbf{0 . 0 0 1}, 6$ & & & & 4,6 \\
\hline & IV: $\mathrm{U}=2334, \mathbf{p}=\mathbf{0 . 0 1 1}, 7$ & IV: $\mathrm{U}=1480, \mathbf{p}=\mathbf{0 . 2 0 3}, 4$ & IV: $\mathrm{U}=1420, \mathbf{p}=\mathbf{0 . 1 2}, 4$ & IV: $\mathrm{U}=1859, \mathrm{p}=0.738,0$ & IV: $\mathrm{U}=1210, \mathbf{p}=\mathbf{0 . 0 1 1}, 9$ \\
\hline & CBW: $\mathrm{U}=861, \mathbf{p}<\mathbf{0 . 0 0 1}, 10$ & CBW: $\mathrm{U}=896, \mathbf{p}<\mathbf{0 . 0 0 1}, 9$ & CBW: $\mathrm{U}=807, \mathbf{p}<\mathbf{0 . 0 0 1}, 9$ & CBW: $\mathrm{U}=1333, \mathbf{p}=\mathbf{0 . 0 2 4}, 7$ & CBW: $U=2483, \mathbf{p}<\mathbf{0 . 0 0 1}, 10$ \\
\hline & $\mathrm{gV}: \mathrm{t}=5.553, \mathbf{p}<\mathbf{0 . 0 0 1}, 10$ & $\mathrm{gV}: \mathrm{t}=4.111, \mathbf{p}<\mathbf{0 . 0 0 1}, 6$ & $\mathrm{gV}: \mathrm{t}=6.94, \mathbf{p}<\mathbf{0 . 0}$ & $\mathrm{gV}: \mathrm{t}=4.474, \mathbf{p}<\mathbf{0 . 0 0 1}, 7$ & $\mathrm{gV}: \mathrm{t}=2.263, \mathbf{p}=\mathbf{0 . 0 3 6}, 5$ \\
\hline & & lV: & & & $3, \mathbf{p}=\mathbf{0}$. \\
\hline & CBW: $\mathrm{U}=670, \mathbf{p}<\mathbf{0 . 0 0 1}, 10$ & CBW: $\mathrm{U}=636, \mathbf{p}<\mathbf{0 . 0 0 1}, 10$ & CBW: $\mathrm{U}=741, \mathbf{p}<\mathbf{0 . 0 0 1}, 10$ & CBW: $U=1427, \mathbf{p}=\mathbf{0 . 0 7 8}, 3$ & CBW: $\mathrm{U}=2878, \mathbf{p}<\mathbf{0}$. \\
\hline & $\mathrm{gV}: \mathrm{t}=6.233, \mathbf{p}<\mathbf{0 . 0 0 1}, 8$ & $\mathrm{gV}: \mathrm{t}=6.979, \mathbf{p}<\mathbf{0 . 0 0 1}, 10$ & $\mathrm{gV}: \mathrm{t}=7.365, \mathbf{p}<\mathbf{0 . 0 0 1}, 10$ & $\mathrm{gV}: \mathrm{t}=4.483, \mathbf{p}<\mathbf{0 . 0 0 1}, 7$ & $\mathrm{gV}: \mathrm{t}=2.079, \mathbf{p}=\mathbf{0 . 0 5 5}, 4$ \\
\hline & lV: $U=1796, p=0.933,2$ & lV: $\mathrm{U}=1724, \mathbf{p}=\mathbf{0 . 8 8 3}, 4$ & $\mathrm{U}=2002, \mathrm{p}=03$ & $\mathrm{lV}: \mathrm{U}=1734, \mathrm{p}=0.9,1$ & lV: $\mathrm{U}=1223, \mathbf{p}=\mathbf{0 . 0 1}$ \\
\hline & $\begin{array}{c}\text { CBW: } \mathrm{U}=1084, \mathbf{p}<\mathbf{0 . 0 0 1}, 9 \\
\mathrm{gV}: \mathrm{t}=4.641, \mathbf{p}<\mathbf{0 . 0 0 1}, 7\end{array}$ & $\begin{array}{c}\text { CBW: } U=1217, \mathbf{p}=\mathbf{0 . 0 0 5}, 8 \\
\text { gV: } t=4.724, \mathbf{p}<\mathbf{0 . 0 0 1 , 8}\end{array}$ & $\begin{array}{c}\text { CBW: } U=1248, \mathbf{p}=\mathbf{0 . 0 0 7}, 9 \\
\text { gV: } t=4.679, \mathbf{p}<\mathbf{0 . 0 0 1 . 8}\end{array}$ & $\begin{array}{c}\text { CBW: } U=2130, \mathbf{p}=\mathbf{0 . 0 6 5}, 3 \\
\mathrm{gV}: \mathrm{t}=-0.555, \mathrm{p}=0.619,0\end{array}$ & $\begin{array}{c}\text { CBW: } U=2663, \mathbf{p}<\mathbf{0 . 0 0 1}, 10 \\
\text { gV: } t=-0.574, \mathbf{p}=\mathbf{0 . 6 1}, 4\end{array}$ \\
\hline & IV: $U=2245, \mathbf{p}=\mathbf{0 . 0}$ & lV: $\mathrm{U}=2348, \mathbf{p}=\mathbf{0 . 0}$ & & IV: $\mathrm{U}=1274, \mathbf{p}=\mathbf{0 . 0}$ & IV: $\mathrm{U}=1323, \mathbf{p}=\mathbf{0 . 0 4 2}, 5$ \\
\hline & CBW: $U=2824, \mathbf{p}<0$ & $\mathrm{CBW}: \mathrm{U}=2755, \mathbf{p}<\mathbf{0}$ & CBW: $\mathrm{U}=2976, \mathbf{p}<$ & $\mathrm{U}=2535, \mathrm{p}$ & $\mathrm{U}=2392, \mathbf{p}=$ \\
\hline & $\mathrm{gV}: \mathrm{t}=3.172, \mathbf{p}=\mathbf{0}$ & $\mathrm{gV}: \mathrm{t}=2.717, \mathbf{p}=\mathbf{0 . 0}$ & $\mathrm{gV}: \mathrm{t}=2.422, \mathbf{p}=\mathbf{0}$ & $\mathrm{t}=0.603, \mathrm{p}=0.593,2$ & $\mathrm{gV}: \mathrm{t}=-3.401, \mathbf{p}=\mathbf{0 . 0 0 2}, 10$ \\
\hline & & lV: $U=2145, p=0.092,0$ & lV: $\mathrm{U}=1707, \mathrm{p}=0.823,2$ & $\mathrm{lV}: \mathrm{U}=1391, \mathbf{p}=\mathbf{0 . 0 8 9}, 4$ & $\mathrm{lV}: \mathrm{U}=2790, \mathbf{p}<\mathbf{0 . 0 0 1}, 9$ \\
\hline
\end{tabular}

TABLE I: Differences in CBW, gV and IV. CBW and IV tests are performed using Mann-Whitney test, and gV is compared using GGLM. The results are reported in the following order: test statistic, p-value and number of consequent thresholds where significant differences were observed. The input frequency is on the vertical facets, and the output frequency is on the horizontal.

cause CS and CBS are influenced by amplitude, while PSI is a pure phase coupling measure. Fraga et al. 31. report an increase of the $\delta-\theta$ and $\delta-\beta$ CFC in AD comparable to our results. This supports the claim that CBS indeed measures some mixture of CFC [21].

\section{Multilayer network analysis}

In order to elucidate the roles of the frequency bands and their coupling, both WFC and CFC, we analyse the CBS networks as multilayer networks with 5 layers representing the traditional frequency bands of EEG.

First, CBW is used to assess the importance of each type of coupling for both local and global communication in the network. Numerical results of comparing CBW are reported in Table $\mathrm{I}$ and visualised in Fig. 6. The in- creased importance of low-frequency-first coupling ( $\delta$ and $\theta$ to the rest) was observed in $\mathrm{AD}$, while the importance of high-frequency-first coupling is decreased compared to HC. This finding confirms the characteristic slowing down of AD signals [6, 7].

Then, $\mathrm{gV}$ is used to assess the vulnerability of information integration of the network to the removal of a coupling type. Numerical results of comparing $\mathrm{gV}$ are reported in Table $\mathrm{I}$ and visualised in Fig. 7. The AD brain networks are significantly more vulnerable as the removal of most coupling types increases $\mathrm{gV}$ except for $\theta-\gamma, \alpha-\gamma, \beta-\beta, \beta-\gamma$ and $\gamma-\beta$ where there is no difference between the groups. Moreover, $\gamma$-couplings show increased $\mathrm{gV}$ in $\mathrm{HC}$, which we suggest to be linked with the decrease in $\gamma$ activity in AD [7. Although mostly slow waves are important in $\mathrm{AD}$ as measured with $\mathrm{CBW}$, the global vulnerability of $\mathrm{AD}$ extends to almost all types 


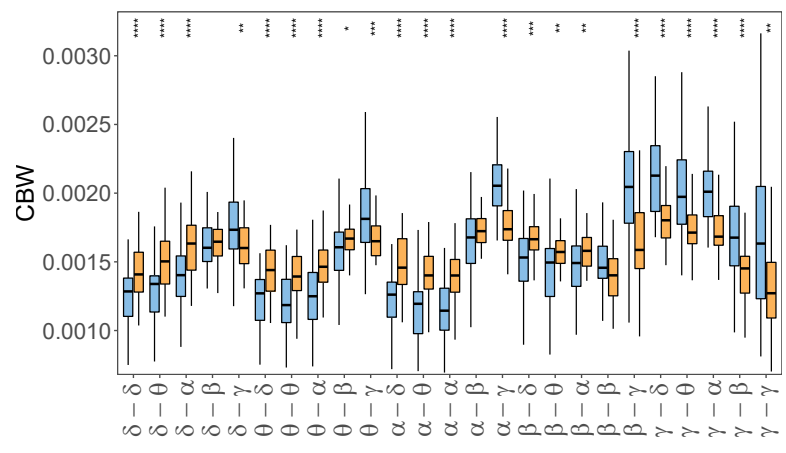

FIG. 6: Importance of each type of frequency coupling of $\mathrm{HC}$ (blue) and $\mathrm{AD}$ (orange) measured by edge betweenness. Significant differences are encoded by asterisks: $\mathrm{p} \leq 0.05$ $(*), \mathrm{p} \leq 0.01(* *), \mathrm{p} \leq 0.001(* * *), \mathrm{p} \leq 0.0001(* * * *)$.

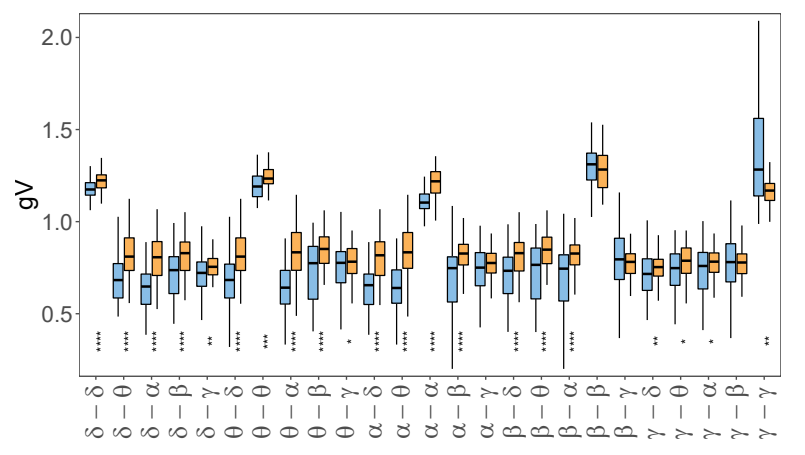

FIG. 7: Global vulnerability of $\mathrm{HC}$ (blue) and AD (orange). Significant differences are encoded by asterisks: $\mathrm{p} \leq 0.05$ $(*), \mathrm{p} \leq 0.01(* *), \mathrm{p} \leq 0.001(* * *), \mathrm{p} \leq 0.0001\left(^{* * * *}\right)$.

of coupling. Interestingly, WFC types cause a generally larger increase in $\mathrm{gV}$, suggesting that while $\mathrm{CFC}$ plays a crucial role in the brain networks, WFC seems to be dominant in the brain networks.

Finally, lV is used to assess the vulnerability of segregation of the network to the removal of certain coupling type. Numerical results of comparing IV are reported in Table 1 and visualised in Fig. 8. This network property seems to be mostly unrelated to the types of coupling,

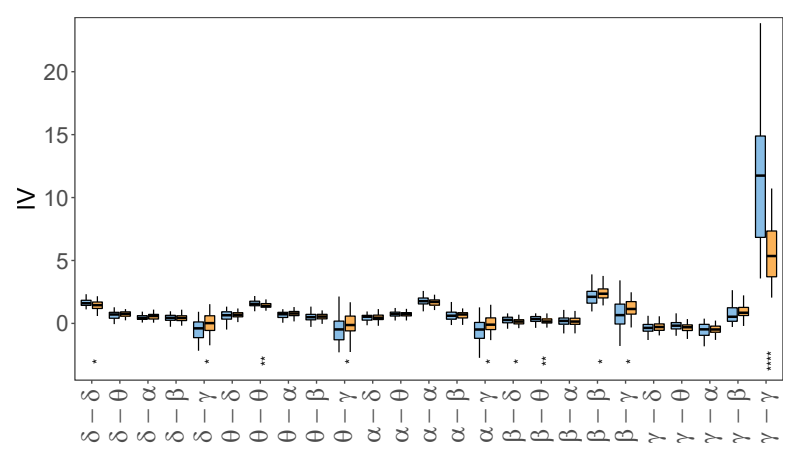

FIG. 8: Local vulnerability of $\mathrm{HC}$ (blue) and AD (orange). Significant differences are encoded by asterisks: $\mathrm{p} \leq 0.05$ $\left.(*), \mathrm{p} \leq 0.01{ }^{(* *}\right), \mathrm{p} \leq 0.001\left(^{* * *}\right), \mathrm{p} \leq 0.0001\left(^{* * * *}\right)$. and we speculate that a mixture of coupling types enables it. The $\gamma-\gamma$ type is the most robustly linked to segregation which fits well with the hypothesis of high-frequency bands being related to local processing. Moreover, this type of coupling is significantly more vulnerable in $\mathrm{HC}$, which is likely related to the loss of $\gamma$ activity in AD [6]. $\delta-\delta$ causes a significant increase of $\mathrm{lV}$ in $\mathrm{HC}$, suggesting that although it is predominantly thought to support long-range connectivity, HC networks use it to some extent for local processing as well, and this role is likely disrupted in $\mathrm{AD}$. On the other hand, $\delta-\gamma, \alpha-\gamma$ and $\beta-\beta$ cause a significant increase in $\mathrm{lV}$ in $\mathrm{AD}$.

\section{Conclusions and Future work}

We have demonstrated that CBS and CS detect similar differences between $\mathrm{AD}$ and $\mathrm{HC}$ networks, but CBS has an advantage over CS by including cross-frequency and nonlinear interactions. We report several significant differences in CFC both globally and on node-level, suggesting that including CFC in a graph-theoretic analysis of brain networks is crucial to obtain a more detailed insight into their structure and function. Furthermore, we show that multilayer network analysis provides a simple yet powerful framework for representing and analysing CFC brain networks. We present a novel approach to elucidate the roles of different frequency components of EEG signals using this framework.

CFC had been suggested to be related to modulatory activity, slow band modulating the activity of fast oscillations. However, it remains unclear why CFC would be increased in $\mathrm{AD}$ and requires further in-depth study.

Next, although (cross-)bispectrum was shown to be a powerful tool to detect various types of WFC and CFC, it is not possible to distinguish between these types of coupling. Therefore, a combination of bispectrum with other types of CFC methods might be a plausible direction for future research.

Furthermore, by relying on traditional frequency bands to define the layers of the networks, our framework might miss some CFC occurring on finer scales, e.g. interaction within one band. However, considering the CFC within only a few bands allows us to construct multilayer networks with a relatively small number of layers. Thus, we argue that relying on the five bands is necessary to introduce the CFC into network analysis without increasing the complexity significantly.

Finally, the presented multilayer network analysis focused only on how dependent or vulnerable the networks are on different types of frequency coupling to enable integration and segregation properties. Although these two properties are hypothesised to be crucial in brain networks, their analysis is not sufficient to elucidate the different functions the frequency couplings might serve in normal brains and how these functions disappear or change during AD.

Moreover, it might be possible to use the multilayer 
networks features to classify the EEG networks automatically. We speculate that including the information about CFC might lead to machine learning models that perform significantly better than models that use only WFCbased features. However, we do not explore this route in this work. Instead, we focus on the relatively novel multilayer network analysis to show the importance of considering CFC in addition to WFC when reconstructing EEG functional connectivity networks.

\section{ACKNOWLEDGEMENT}

The original data collection was funded by a grant from the Alzheimer's Research UK (ARUK-PPG20114B-25). The views expressed are those of the author(s) and not necessarily those of the NHS, the NIHR or the Department of Health.
[1] Christina Patterson et al. World alzheimer report 2018. 2018.

[2] Leide C. dos Santos Picanco, Priscilla F. Ozela, Maiara de Fatima de Brito Brito, Abraao A. Pinheiro, Elias C. Padilha, Francinaldo S. Braga, Carlos H. T. de Paula da Silva, Cleydson Breno Rodrigues dos Santos, Joaquín M. C. Rosa, and Lorane Izabel da Silva Hage-Melim. Alzheimer's disease: A review from the pathophysiology to diagnosis, new perspectives for pharmacological treatment. Current Medicinal Chemistry, 25(26):3141-3159, -08-01 2018.

[3] Michela Pievani, Willem de Haan, Tao Wu, William W Seeley, and Giovanni B Frisoni. Functional network disruption in the degenerative dementias. The Lancet Neurology, 10(9):829-843, 2011.

[4] Thomas König, L Prichep, Thomas Dierks, Daniela Hubl, LO Wahlund, ER John, and V Jelic. Decreased eeg synchronization in alzheimer's disease and mild cognitive impairment. Neurobiology of aging, 26(2):165-171, 2005.

[5] Xavier Delbeuck, Martial Van der Linden, and Fabienne Collette. Alzheimer'disease as a disconnection syndrome? Neuropsychology review, 13(2):79-92, 2003.

[6] Jaeseung Jeong. Eeg dynamics in patients with alzheimer's disease. Clinical neurophysiology, 115(7):1490-1505, 2004.

[7] Parham Ghorbanian, David M Devilbiss, Terry Hess, Allan Bernstein, Adam J Simon, and Hashem Ashrafiuon. Exploration of eeg features of alzheimer's disease using continuous wavelet transform. Medical $\&$ biological engineering ES computing, 53(9):843-855, 2015.

[8] Justin Dauwels, François Vialatte, Toshimitsu Musha, and Andrzej Cichocki. A comparative study of synchrony measures for the early diagnosis of alzheimer's disease based on eeg. NeuroImage, 49(1):668-693, 2010.

[9] O. Vyšata, M. Vališ, A. Procházka, R. Rusina, and L. Pazdera. Linear and nonlinear eeg synchronization in alzheimer's disease. Neurophysiology, 47(1):46-52, 2015.

[10] Claudio Babiloni, Roberta Lizio, Nicola Marzano, Paolo Capotosto, Andrea Soricelli, Antonio Ivano Triggiani, Susanna Cordone, Loreto Gesualdo, and Claudio Del Percio. Brain neural synchronization and functional coupling in alzheimer's disease as revealed by resting state eeg rhythms. International Journal of Psychophysiology, 103:88-102, 2016.

[11] A Kabbara, H Eid, W El Falou, M Khalil, F Wendling, and M Hassan. Reduced integration and improved segregation of functional brain networks in alzheimer's disease. Journal of neural engineering, 15(2):026023, 2018.
[12] Zhengjia Dai, Qixiang Lin, Tao Li, Xiao Wang, Huishu Yuan, Xin Yu, Yong He, and Huali Wang. Disrupted structural and functional brain networks in alzheimer's disease. Neurobiology of aging, 75:71-82, 2019.

[13] Kaustubh Supekar, Vinod Menon, Daniel Rubin, Mark Musen, and Michael D Greicius. Network analysis of intrinsic functional brain connectivity in alzheimer's disease. PLoS Comput Biol, 4(6):e1000100, 2008.

[14] Viktor Jirsa and Viktor Müller. Cross-frequency coupling in real and virtual brain networks. Frontiers in computational neuroscience, 7:78, 2013.

[15] G Adler, S Brassen, and A Jajcevic. Eeg coherence in alzheimer's dementia. Journal of neural transmission, 110(9):1051-1058, 2003.

[16] Dong-Hwa Jeong, Young-Do Kim, In-Uk Song, Yong-An Chung, and Jaeseung Jeong. Wavelet energy and wavelet coherence as eeg biomarkers for the diagnosis of parkinson's disease-related dementia and alzheimer's disease. Entropy, 18(1):8, 2016.

[17] Lihui Cai, Xile Wei, Jiang Wang, Haitao Yu, Bin Deng, and Ruofan Wang. Reconstruction of functional brain network in alzheimer's disease via cross-frequency phase synchronization. Neurocomputing, 314:490-500, 2018.

[18] Jérémy Guillon, Yohan Attal, Olivier Colliot, Valentina La Corte, Bruno Dubois, Denis Schwartz, Mario Chavez, and F De Vico Fallani. Loss of brain inter-frequency hubs in alzheimer's disease. Scientific reports, 7(1):1-13, 2017.

[19] Matthew J Brookes, Prejaas K Tewarie, Benjamin AE Hunt, Sian E Robson, Lauren E Gascoyne, Elizabeth B Liddle, Peter F Liddle, and Peter G Morris. A multi-layer network approach to meg connectivity analysis. Neuroimage, 132:425-438, 2016.

[20] Fei He and Yuan Yang. Nonlinear system identification of neural systems from neurophysiological signals. Neuroscience, 458:213-228, 2021.

[21] Viktor Jirsa and Viktor Müller. Cross-frequency coupling in real and virtual brain networks. Frontiers in computational neuroscience, 7:78, 2013.

[22] Ruofan Wang, Jiang Wang, Shunan Li, Haitao Yu, Bin Deng, and Xile Wei. Multiple feature extraction and classification of electroencephalograph signal for alzheimers' with spectrum and bispectrum. Chaos: An Interdisciplinary Journal of Nonlinear Science, 25(1):013110, 2015.

[23] Naghmeh Mahmoodian, Axel Boese, Michael Friebe, and Javad Haddadnia. Epileptic seizure detection using crossbispectrum of electroencephalogram signal. seizure, 66:4$11,2019$.

[24] Rajamanickam Yuvaraj, U Rajendra Acharya, and Yuki Hagiwara. A novel parkinson's disease diagnosis index 
using higher-order spectra features in eeg signals. Neural Computing and Applications, 30(4):1225-1235, 2018.

[25] Daniel J. Blackburn, Yifan Zhao, Matteo De Marco, Simon M. Bell, Fei He, Hua-Liang Wei, Sarah Lawrence, Zoe C. Unwin, Michelle Blyth, and Jenna Angel. A pilot study investigating a novel non-linear measure of eyes open versus eyes closed eeg synchronization in people with alzheimer's disease and healthy controls. Brain sciences, 8(7):134, 2018.

[26] James Theiler, Stephen Eubank, André Longtin, Bryan Galdrikian, and J Doyne Farmer. Testing for nonlinearity in time series: the method of surrogate data. Physica D: Nonlinear Phenomena, 58(1-4):77-94, 1992.

[27] Alain Barrat, Marc Barthelemy, Romualdo PastorSatorras, and Alessandro Vespignani. The architecture of complex weighted networks. Proceedings of the national academy of sciences, 101(11):3747-3752, 2004.

[28] Michelle Girvan and Mark EJ Newman. Community structure in social and biological networks. Proceedings of the national academy of sciences, 99(12):7821-7826, 2002.

[29] Shuting Sun, Xiaowei Li, Jing Zhu, Ying Wang, Rong La, Xuemin Zhang, Liuqing Wei, and Bin Hu. Graph theory analysis of functional connectivity in major depression disorder with high-density resting state eeg data. IEEE Transactions on Neural Systems and Rehabilitation Engineering, 27(3):429-439, 2019.

[30] Vito Latora and Massimo Marchiori. Vulnerability and protection of infrastructure networks. Physical Review E, 71(1):015103, 2005.

[31] Francisco J Fraga, Tiago H Falk, Paulo AM Kanda, and Renato Anghinah. Characterizing alzheimer's disease severity via resting-awake eeg amplitude modulation analysis. PloS one, 8(8):e72240, 2013. 